\title{
PUBLICAÇÕES CIENTÍFICAS DO LABORATÓRIO DE EXPERIMENTAÇÃO REMOTA - REXLAB: UMA REVISÃO SISTEMÁTICA
}

\author{
LAS PUBLICACIONES CIENTÍFICAS DE \\ LABORATORIO DE EXPERIMENTACIÓN A DISTANCIA - \\ REXLAB: UNA REVISIÓN SISTEMÁTICA
}

\author{
Janine De Lavechia* \\ Juarez Bento da Silva** \\ Fernando José Spanhol***
}

\begin{abstract}
RESUMO:
Introdução: As Tecnologias de Informação e Comunicação TIC estão a cada dia permitindo o aperfeiçoamento em todos os níveis das instituições, proporcionando em um contexto geral maior comunicação, e assim possibilitando a criação de novos conhecimentos. E com o surgimento do Laboratório de Experimentação Remota RExLab, que possibilita ao aluno realizar experimentos, viabiliza a construção de novos conhecimentos.

Objetivo: Identificar e apresentar as NTIC (Novas Tecnologias da Informação e Comunicação) pesquisadas no laboratório e explicitadas nas publicações dos integrantes vinculados ao RExLab.

Metodologia: Neste artigo foi realizada uma pesquisa sistemática sobre os artigos publicados no RExLab, e situado na Universidade Federal de Santa Catarina, Araranguá. E os artigos com maiores citações, foram apresentadas as NTIC utilizadas em cada pesquisa.

Resultados: Foi possível identificar que uma grande maioria dos artigos publicados estava relacionada em questões da experimentação remota através de uso de dispositivos móveis, e que hoje é de grande acesso a quase todos os alunos.

Conclusões: Foi possível analisar que o maior objetivo das pesquisas publicadas é a realização da democratização e a disseminação do conhecimento através de seus experimentos. Pois são projetos que podem estar aplicados em todos os níveis de ensino e também tanto em escolas públicas como em escolas privadas. Com isso, há muitas TIC que estão inseridas nos projetos e que possuem como propósito o auxílio à educação.
\end{abstract}

Palavras-chave: Publicações. RExLab. NTIC.

*Mestranda do programa do Pós-Graduação em Tecnologia da Informação e Comunicação UFSC. E-mail: janinedelavechia@hotmail.com

**Doutor em Engenharia e Gestão do Conhecimento - UFSC. Email: juarez.silva@ufsc.br

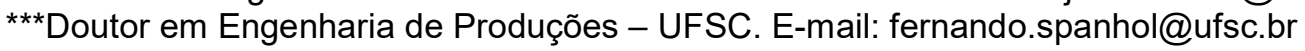


Janine De Lavechia, Juarez Bento da Silva, Fernando José Spanhol

Publicações científicas do laboratório de experimentações remota - RExLab: uma revisão sistemática:

\section{INTRODUÇÃO}

A sociedade vem sofrendo grandes impactos com a chegada das tecnologias para facilitar o cotidiano, em diferentes áreas como a industrial e a educacional. Instituições de ensino acabam perdendo vantagem, pois muitas delas não possuem uma infraestrutura adequada para compartilhar 0 conhecimento de forma aplicada com os alunos. Não possuem laboratórios físicos para realização de experimentos, não possibilitando assim a construção do conhecimento pelo aluno de forma prática.

Para Capellini e Conrado (2009, p. 1) "as dificuldades de aprendizagem podem ser entendidas como obstáculos ou barreiras encontradas por alunos durante o período de escolarização, referentes à captação ou à assimilação dos conteúdos propostos". Sendo que essas dificuldades podem ser duradouras ou passageiras, chegando até levar alunos ao abandono da escola, à reprovação, ao baixo rendimento, e até ao atraso no tempo de aprendizagem.

Com isso, surgiram os Laboratórios Remotos com o intuito de complementar e facilitar o entendimento dos alunos em relação a algumas disciplinas (através de experimentos) que possuem maior grau de dificuldade, com relação a conteúdos de ensino, que na teoria não se torna claro.

Laboratórios Remotos diferem de simulações ou laboratórios virtuais. No laboratório remoto há maior aproximação do resultado, pois utilizam experimentos reais. Além de tudo, o acesso se torna mais fácil, pois necessita apenas de acesso à internet, e várias escolas podem estar acessando ao mesmo tempo (SIMÃO et al., 2013), tornando assim uma forma econômica de acesso ao laboratório.

O Laboratório de Experimentação Remota - RExLab foi criado em 1997 no Departamento de Informática e de Estatística do Centro Tecnológico da Universidade Federal de Santa Catarina (INE/CTC/UFSC), trazendo para o Brasil desenvolvimento tecnológico para a realização de experimentos de laboratório feitos remotamente, via Internet. O objetivo principal é compartilhar recursos entre instituições de ensino, reduzindo assim o custo na educação e no desenvolvimento científico e tecnológico, pois os investimentos em 
Janine De Lavechia, Juarez Bento da Silva, Fernando José Spanhol

Publicações científicas do laboratório de experimentações remota - RExLab: uma revisão sistemática:

equipamentos e a manutenção de um laboratório que permite realizar esses experimentos são altos. O RExLab está vinculado aos Programas de PósGraduação em Tecnologias da Informação e Comunicação (PPGTIC) e Engenharia e Gestão do Conhecimento (PPGEGC) (LATTES, 2016), e possui diversas parcerias com outras instituições e em diferentes países.

Ao todo são 24 (vinte e quatro) integrantes que realizam atividades no RExLab UFSC - Campus Araranguá, divididos em: 1 Supervisor; 2 Coordenadores; 11 na Equipe Técnica e 10 na Equipe Pedagógica. Grande parte dos envolvidos atua a certo tempo no laboratório e desenvolvem pesquisas e realizam publicações referentes à suas pesquisas.

Para Brofman (2012) as publicações científicas divulgam a pesquisa para a comunidade, e permitem que outros pesquisadores possam utilizá-las e avaliá-las sob outras visões. Brofman (2012, p. 419) afirma que "é por meio de uma publicação científica que a sociedade toma conhecimento dos resultados de um trabalho de pesquisa e o que este representa para a coletividade".

Este estudo tem como objetivo de identificar e apresentar as NTICs (Novas Tecnologias da Informação e Comunicação) pesquisadas no laboratório e explicitadas nas publicações dos integrantes vinculados ao RExLab. Para assim, identificarmos o que está sendo produzido e que servirá para futuramente aplicar técnicas de Gestão do Conhecimento no contexto do laboratório.

\section{NOVAS TENOLOGIAS DE INFORMAÇÃO E COMUNICAÇÃO}

As Novas Tecnologias da Informação e Comunicação (NTIC) provocaram mudanças nas últimas décadas, onde possibilitou que pessoas pudessem conversar e trocar informações em tempo real com pessoas do outro lado do mundo, onde o grande impacto foi com a chegada da internet. Cruz (1998) já havia descrito sobre as TIC, caracterizando-as como um conjunto de dispositivos individuais - hardware, software - ou qualquer outra tecnologia que faça parte ou que contenha informação.

Assim Crispim (2013, p. 1) apresenta o conceito de TIC: 
Janine De Lavechia, Juarez Bento da Silva, Fernando José Spanhol

Publicações científicas do laboratório de experimentações remota - RExLab: uma revisão sistemática:

As Tecnologias da Informação e Comunicação (TIC) são um conjunto de recursos tecnológicos integrados entre si, que proporcionam, por meio das funções tecnológicas, a simplificação da comunicação nos processos de negócios, da pesquisa científica, de ensino e aprendizagem (...).

Gomes e Dourado (2013, p. 3) conceituam as NTICs:

Normeiam-se de Novas Tecnologias de Informação e Comunicação (NTICs) as tecnologias e métodos para comunicar surgidas no contexto da Revolução Informacional, desenvolvidas gradativamente desde a segunda metade da década de 1970 e, principalmente, nos anos de 1990. A maioria delas caracteriza-se por agilizar, horizontalizar e tornar menos físico o conteúdo da comunicação, por meio da digitalização e da comunicação em redes para a captação, transmissão e distribuição das informações (texto, imagem estática, vídeo e som). Considera-se que o advento destas novas tecnologias possibilitou o surgimento da "sociedade da informação".

Sendo assim, as TIC existiram desde a antiguidade, com o descobrimento da escrita, que possibilitou a troca de informações entre os povos. E as NTICs surgiram com a chegada das TIC digitais, possibilitando a realização da comunicação através da rede.

Atualmente as NTICs estão por toda a parte, TV, computadores, webcam, máquina fotográfica, internet, e-mail, smartphones, entre outros aparelhos. Assim Takahashi (2000, p. 29) informa sobre a presença e influência das Novas Tecnologias de Informação e Comunicação afirmando que:

Assistir à televisão, falar ao telefone, movimentar a conta no terminal bancário e pela Internet, verificar multas de trânsito, comprar discos, trocar mensagens com o outro lado do planeta, pesquisar e estudar são hoje atividades cotidianas, no mundo inteiro e no Brasil. Rapidamente nos adaptamos a essas novidades e passamos - em geral, sem uma percepção clara nem maiores questionamentos - a viver na Sociedade da Informação, uma nova era em que a informação flui a velocidades e em quantidades há apenas poucos anos inimagináveis, assumindo valores sociais e econômicos fundamentais.

Para Silva (2006) a introdução das NTICs possibilitou a comunicação entre estudantes de diferentes lugares do país e do mundo, consequentemente 
Janine De Lavechia, Juarez Bento da Silva, Fernando José Spanhol

Publicações científicas do laboratório de experimentações remota - RExLab: uma revisão sistemática:

maior acesso a informação on-line e o envolvimento em projetos colaborativos, onde a maioria dos participantes não se conhece pessoalmente.

Com isso, os professores devem receber um treinamento para realizar essa inclusão das tecnologias no ambiente educacional. As NTICs desenvolvidas no RExLab são um exemplo e que o professor pode estar apresentado e utilizando com os alunos, onde os mesmos irão verificar na prática, a teoria que o professor repassou na sala de aula.

\section{REXLAB}

Laboratórios remotos oferecem o acesso a equipamentos, bancadas e experimentos do laboratório por meio de um computador conectado à Internet (CARDOSO; TAKAHASHI, 2011). São utilizados para aulas experimentais, onde os alunos poderão construir seus conhecimentos, através da prática.

Lima, Rochadel e Silva (2013, p. 3) afirmam que:

A experimentação remota se utiliza de elementos do ensino eletrônico (e-learning) no ensino presencial para disponibilizar experimentos físicos à distância dentro de uma aula que os alunos não usufruem destes recursos ou são impossibilitados de frequentar espaços laboratoriais.

Laboratórios de experimentação remota são acessíveis 24 horas por dia, e 7 dias por semana. Estes laboratórios podem resolver problemas como o congestionamento, a falta de espaço e a falta de laboratórios, possibilitando a realização de experimentos em casa. Experiências remotas permitem uma aprendizagem mais flexível no tempo, lugar, ritmo, com acesso a um grande número de experimentos e redução de custos (SILVA, 2015).

A Figura 1 ilustra a estrutura de um modelo para a criação de um RExLab. Silva (2006) salienta que os alunos acessam via o protocolo TCP/IP (principal protocolo de envio e recebimento de dados via internet) os equipamentos ou hardware e os programas, e desta forma podem controlar e observar a execução dos experimentos através de um Webcam ou outro meio. 
Janine De Lavechia, Juarez Bento da Silva, Fernando José Spanhol

Publicações científicas do laboratório de experimentações remota - RExLab: uma revisão sistemática:

Figura 1 -Um modelo básico para um RExLab

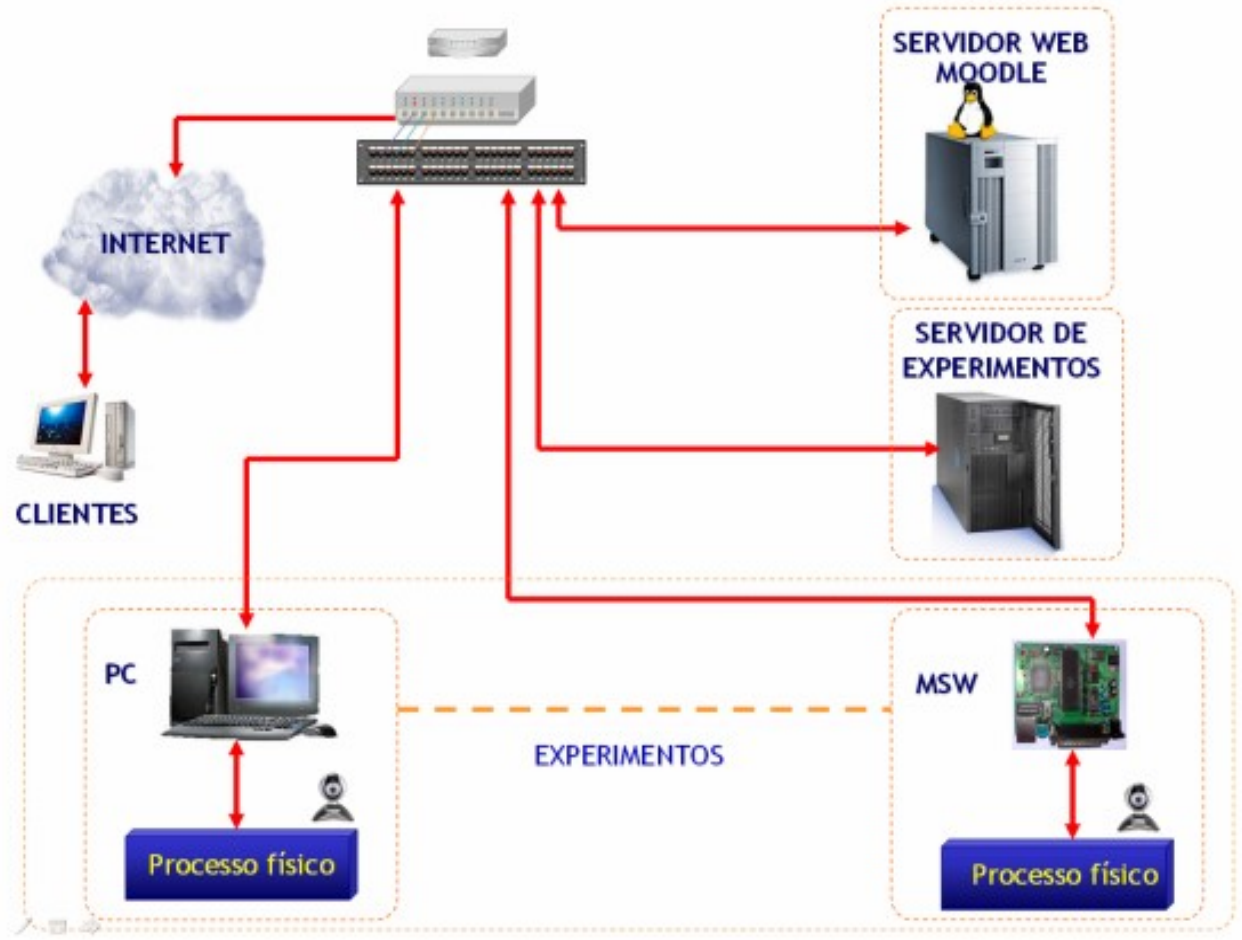

Fonte: Silva (2006)

O RExLab é um laboratório que explora diversas níveis do ensino, onde se está pesquisando e implementando já faz vários anos a aplicação das NTICs no ensino com a perspectiva de incorporar atividades experimentais em forma remota e para instâncias complementares a distância (SILVA, 2006).

Silva (2006, p. 164) explica sobre a área de Circuitos Digitais:

$\mathrm{Na}$ área de Circuitos Digitais são utilizados dispositivos micro controlados onde os estudantes podem gravar e testar as aplicações desenvolvidas e acionar outros dispositivos elétricos, tais como displays, relés, motores, ventiladores, lâmpadas. O propósito deste experimento é disponibilizar um modelo didático orientado a promover o raciocínio qualitativo, a conceitualização dos fenômenos e a criatividade reforçando os conhecimentos teóricos e estimulando-os a encontrar respostas.

Atualmente o RExLab conta no total com 12 experimentos online, que podem ser acessados através do site (http://relle.ufsc.br/) de qualquer lugar, a qualquer hora, para verificação e apreciação de resultados. Como há um grande número de pessoas envolvidas, e que estão constantemente 
Janine De Lavechia, Juarez Bento da Silva, Fernando José Spanhol

Publicações científicas do laboratório de experimentações remota - RExLab: uma

revisão sistemática:

pesquisando e realizando publicações, realizou-se uma pesquisa para identificar as NTICs que estão envolvidas no estudo sobre a Experimentação Remota.

\section{PROCEDIMENTOS METODOLÓGICOS}

O método utilizado para a construção deste artigo fundamenta-se em uma pesquisa descritiva e com abordagem qualitativa. A técnica utilizada para a coleta de dados partiu de uma busca sistêmica sobre as publicações realizadas pelo grupo de pesquisadores do RExLab, a fim de identificar as NTICs utilizadas por eles. Posteriormente foram realizados alguns procedimentos bibliométricos com a intenção de identificar a produção por área, período, para que seja possível situar a produção científica do grupo. Para Silva e Spanhol (2013, p. 10) "esse método de análise, pode ser utilizado para a construção do 'estado da arte' de uma determinada área ou assunto".

Desta maneira estabeleceu a seguinte pergunta da pesquisa: Quais foram as publicações do RExLab que abordam as Novas Tecnologias da Informação e Comunicação?

O próximo passo foi identificar quais publicações seriam analisadas. A escolha foi pelos artigos que estavam dispostos na base de publicações do RExLab.

Os resultados consistem na coleta, análise, apresentação e na interpretação dos dados extraídos dos artigos e serão apresentados a seguir. Definiu-se então que a coleta de dados foi predominantemente qualitativa, e a análise foi realizada por meio das técnicas de análise de conteúdo do resumo.

\section{ANÁLISE DAS RELAÇÕES}

Através da análise quantitativa, foi possível identificar ao total 31 artigos publicados na página do RExLab no site http://rexlab.ufsc.br/publications - que teve início em 2007, com uma publicação no periódico InternationalJournalof 
Janine De Lavechia, Juarez Bento da Silva, Fernando José Spanhol

Publicações científicas do laboratório de experimentações remota - RExLab: uma revisão sistemática:

Online Engineering (iJOE). Neste primeiro artigo de 2007, com o título: "A ScaleModelof a GrainStorageTorage Tower Equippedwith Micro Web Servers", onde tem o objetivo de apresentar um modelo de um silo para a secagem e aeração de grãos de cereais com o monitoramento e controle fornecidos por um Servidor Web, permitindo armazenar todos os parâmetros em uma base de dados para análise posterior, de preferência feito remotamente. Esse modelo já teria o objetivo de apoiar a instalação de experimentação na educação tecnológica.

No contexto geral das publicações realizou-se uma tabulação das palavras-chaves, e com isso foi possível identificar que a grande maioria dos artigos trazia quase sempre a palavra "Remote Experiments" ocorrendo no total de 31 artigos, em 17 aparecem como palavra-chave, e seguido pelas palavras "Mobile Devices" e "E-learning" com 8 e 7 aparições nas palavras-chave respectivamente, como mostra a Figura 2 a seguir.

Figura 2 - Predominância das palavras-chaves

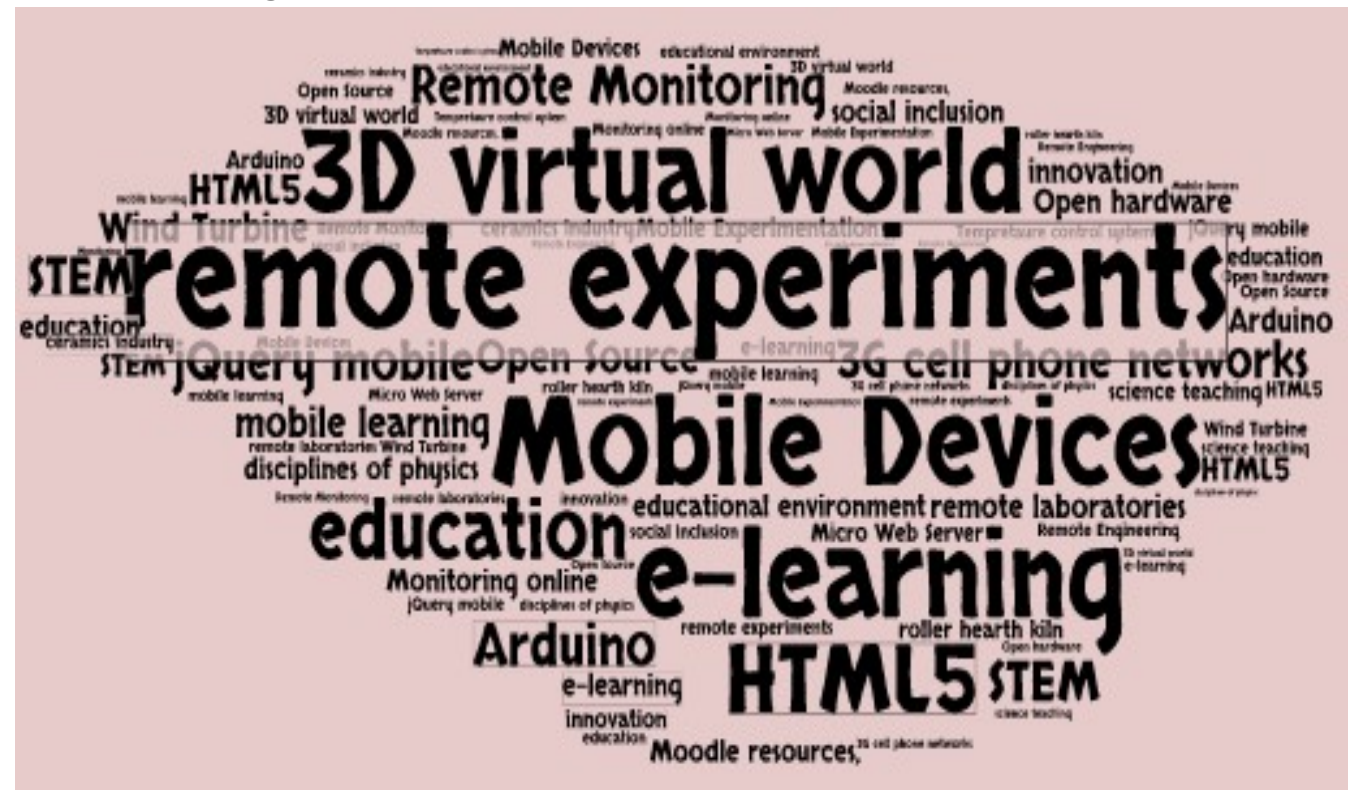

Fonte: Autora (2016)

Todas as palavras-chaves encontradas podem ser consideradas NTICs, pois são termos que surgiram há pouco tempo, e estão imersas no ambiente 
Janine De Lavechia, Juarez Bento da Silva, Fernando José Spanhol

Publicações científicas do laboratório de experimentações remota - RExLab: uma

revisão sistemática:

tecnológico, pois precisam de mecanismos voltados á tecnologia para sua existência.

Pode-se analisar que a palavra "Remote Laboratories" aparece em apenas dois artigos, sendo que é uma palavra que remete ao laboratório em si e tem uma grande relevância ao grupo. Ou seja, a experimentação remota está atrelada em diversas outras áreas, como no ensino de física, nos silos de secagem de grãos, aplicada em dispositivos móveis, e com isso não trouxeram tantos resultados voltados a laboratórios remotos.

Como em todos os artigos existem aplicações de NTICs, montou-se uma tabela ilustrando os artigos que possuem maiores números de citações, pois são os mais impactantes e relevantes para a pesquisa, pois conforme afirma Guedes e Borschiver (2005, p. 11) "artigos de periódicos citados mais frequentemente são mais relevantes do que artigos menos citados". Os artigos estão apresentados na Tabela 1 a seguir, ordenados por ano de publicação:

Tabela 1 - Artigos selecionados com citações e tecnologias

\begin{tabular}{|c|c|c|c|c|c|}
\hline \multirow{2}{*}{ Id } & \multicolumn{5}{|l|}{ Tabela de Artigos } \\
\hline & Título & Autores & Ano & Cit & NTICs \\
\hline 1 & $\begin{array}{l}\text { Adaptation Model of Mobile } \\
\text { Remote Experimentation for } \\
\text { Elementary Schools }\end{array}$ & $\begin{array}{lll}\text { J. B. Silva, } & \text { W. } \\
\text { Rochadel, J. } & \text { P. } \\
\text { Simão, } & \text { A. } & \text { S. } \\
\text { Fidalgo } & & \end{array}$ & 2014 & 5 & $\begin{array}{l}\text { Dispositivos móveis } \\
\text { experiências remotas }\end{array}$ \\
\hline 2 & $\begin{array}{l}\text { Extending access to remote } \\
\text { labs from mobile devices in } \\
\text { educational contexts }\end{array}$ & 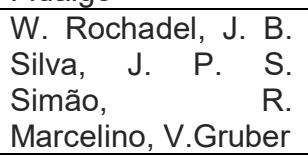 & 2013 & 10 & $\begin{array}{l}\text { Dispositivos móveis e } \\
\text { características do HTML5 e o } \\
\text { jQuery Mobile }\end{array}$ \\
\hline 3 & $\begin{array}{l}\text { Technology PLC - Power Line } \\
\text { Communication, Used in } \\
\text { Monitoring Systems Online }\end{array}$ & $\begin{array}{lll}\text { V. Gruber, } & \text { R. } \\
\text { Marcelino, J. } & \text { B. } \\
\text { Silva } & & \\
\end{array}$ & 2013 & 5 & $\begin{array}{l}\text { PLC (Power } \\
\text { Communication) }\end{array}$ \\
\hline 4 & $\begin{array}{l}\text { Uso de dispositivos móveis } \\
\text { para acesso a Experimentos } \\
\text { Remotos na Educação Básica }\end{array}$ & $\begin{array}{lll}\text { J. B. Silva, } & \text { W. } \\
\text { Rochadel, J. } & \text { P. } \\
\text { Simão, } & \text { A. } & \text { S. } \\
\text { Fidalgo } & & \\
\end{array}$ & 2013 & 3 & $\begin{array}{l}\text { Dispositivos móveis } \\
\text { experiências remotas }\end{array}$ \\
\hline 5 & $\begin{array}{l}\text { Utilização de Experimentação } \\
\text { Remota Móvel no Ensino } \\
\text { Médio }\end{array}$ & $\begin{array}{l}\text { J. P. S. Simão, J. P. } \\
\text { C. Lima, } \\
\text { Rochadel, J. } \\
\text { Silva }\end{array}$ & 2013 & 3 & $\begin{array}{l}\text { Dispositivos móveis } \\
\text { experiências remotas }\end{array}$ \\
\hline 6 & $\begin{array}{l}\text { Utilization of NICTs Applied to } \\
\text { Mobile Devices }\end{array}$ & $\begin{array}{l}\text { J. B. Silva, W. } \\
\text { Rochadel, } \\
\text { Marcelino, V.Gruber }\end{array}$ & 2013 & 12 & $\begin{array}{l}\text { Moodle, mundos virtuais } 3 \mathrm{D} \text { e } \\
\text { experiências remotas }\end{array}$ \\
\hline 7 & $\begin{array}{l}\text { 3D virtual worlds using open } \\
\text { source platform and integrated } \\
\text { remote experimentation }\end{array}$ & $\begin{array}{l}\text { R. Marcelino, J. B. } \\
\text { Silva, V. Gruber, S. } \\
\text { M. S.Bilessimo }\end{array}$ & 2012 & 3 & $\begin{array}{l}\text { Experimentação remota e } \\
\text { ambientes virtuais 3D }\end{array}$ \\
\hline 8 & $\begin{array}{l}\text { New Technologies for } \\
\text { Information and } \\
\text { Communication, PWM Remote } \\
\text { Experimenting and 3G } \\
\text { Networks as Teaching Support }\end{array}$ & $\begin{array}{ll}\text { J. B. Silva, } & \text { V. } \\
\text { Gruber, } & \text { R. } \\
\text { Marcelino } & \end{array}$ & 2012 & 4 & $\begin{array}{l}\text { Rede de telefonia celular } \\
\text { 3Geexperiências remotas }\end{array}$ \\
\hline
\end{tabular}


Janine De Lavechia, Juarez Bento da Silva, Fernando José Spanhol

Publicações científicas do laboratório de experimentações remota - RExLab: uma revisão sistemática:

\begin{tabular}{|c|c|c|c|c|c|}
\hline \multirow{2}{*}{ Id } & \multicolumn{5}{|l|}{ Tabela de Artigos } \\
\hline & Título & Autores & Ano & Cit & NTICs \\
\hline 9 & $\begin{array}{ll}\text { Utilization of } & \text { remote } \\
\text { experimentation in mobile } \\
\text { devices for education }\end{array}$ & $\begin{array}{l}\text { W. Rochadel, S. P. } \\
\text { Silva, J. B. Silva, T. } \\
\text { D. Luz, G. R. Alves }\end{array}$ & 2012 & 6 & $\begin{array}{l}\text { Experimentação remota e } \\
\text { dispositivos móveis }\end{array}$ \\
\hline 10 & $\begin{array}{l}\text { Utilização de NTIC's Aplicadas } \\
\text { a Dispositivos Móveis. }\end{array}$ & $\begin{array}{ll}\text { J. B. Silva, } & \text { W. } \\
\text { Rochadel, } & \text { R. } \\
\text { Marcelino. } & \end{array}$ & 2012 & 8 & $\begin{array}{l}\text { Moodle, mundos virtuais } 3 \mathrm{D} \text { e } \\
\text { experimentação remota. }\end{array}$ \\
\hline 11 & $\begin{array}{l}\text { Model for remote data } \\
\text { acquisition and monitoring } \\
\text { integrating social media, } \\
\text { NTIC's and } 3 G \text { cell phone } \\
\text { Networks applied to monitoring } \\
\text { small wind turbine }\end{array}$ & $\begin{array}{l}\text { V. Gruber, L. } \\
\text { Schaeffer, J. B. } \\
\text { Silva, M. T. Restivo }\end{array}$ & 2011 & 3 & $\begin{array}{l}\text { Rede de telefonia celular } 3 G \\
\text { e as redes sociais }\end{array}$ \\
\hline 12 & $\begin{array}{l}\text { Extended Immersive Learning } \\
\text { Environment: A Hybrid } \\
\text { Remote/Virtual Laboratory }\end{array}$ & $\begin{array}{l}\text { R. Marcelino; Silva, } \\
\text { J. B. Silva; G. R. } \\
\text { Alves, }\end{array}$ & 2010 & 6 & $\begin{array}{l}\text { Mundos virtuais 3D, } \\
\text { aprendizagem e sistemas de } \\
\text { gestão de conteúdos, } \\
\text { experiências remotas, e } \\
\text { espaços de aprendizagem } \\
\text { colaborativa }\end{array}$ \\
\hline
\end{tabular}

Fonte: Autora (2016)

Os dois primeiros trabalhos identificados possuem o mesmo objetivo principal, o uso de dispositivos móveis na educação. No trabalho de Silva et al. (2014); Silva et al. (2013); Simão et al. (2013)(artigos da Tabela 1 - 1, 4, 5 respectivamente), traz um projeto-piloto com o uso das NTICs através dos dispositivos móveis e experimentação remota. Realizado o projeto em escola de educação básica da rede pública de ensino, onde os alunos teriam acesso ao conteúdo educacional e realizariam os experimentos, assim melhorariam a qualidade de experiências de aprendizagem e ampliariam a aprendizagem para além do ambiente escolar tradicional. O projeto traz a arquitetura totalmente em recursos de software de código aberto e hardware que incluem o sistema de gestão de aprendizagem (Moodle), o software aplicativo RExMobile e os experimentos remotos desenvolvidos pelo RExLAb.

Outro estudo que traz a utilização de experimentação remota usando dispositivos móveis nas áreas de ciência é o deRochadelet al. (2012)(artigo 9 da Tabela 1) onde os autores também pretenderam aplicar, porém para uma escola primária,com a finalidade de desenvolver práticas que ajudarão noprocesso de assimilação das matérias ensinadas na sala de aula.

No artigo de Rochadel et al. (2013)(artigo 2 da Tabela 1) também é apresentado uma forma de utilizar experiências remotas através de dispositivos móveis, melhorando o envolvimento dos alunos nativos digitais. Neste trabalho é usada a extensão com características do HTML5 ("é um conjunto maior de 
Janine De Lavechia, Juarez Bento da Silva, Fernando José Spanhol

Publicações científicas do laboratório de experimentações remota - RExLab: uma revisão sistemática:

tecnologias que permite o desenvolvimento de aplicações e web sites mais diversos e poderosos" (MACALHA, 2016)) e o jQuery Mobile ("é uma estrutura efetiva e unificada para criar aplicativos da web móveis" (ORTIZ, 2016)), que permitem 0 acesso as experiências de diferentes sistemas operacionais através do browser ou aplicações nativas. Como resultado, os usuários têm um modo de interação mais rica com os experimentos, o que inclui acesso a partir de dispositivos como smartphones.

Seguindo neste mesmo tema de integrar experimentação remota e dispositivos móveis, os artigos deSilva et al. (2013) e Silva, Rochadel, Marcelino (2012) (artigos 6 e 10, da Tabela 1) trazem o ingresso dos mundos virtuais $3 D$ na experimentação remota com um sistema de gestão de aprendizagem (Moodle). Onde foram aplicadas as NTICs nos dispositivos móveis, proporcionando aos estudantes um acesso mais rápido a informações para continuar seus estudos de tecnologia e engenharia. No trabalho de Marcelino et al. (2012) (artigo 7 da Tabela I) também apresenta a aplicação experimentação remota e mundos virtuais 3D em processo ensinoaprendizagem nas áreas de exatas ciência-física. Acessando o mundo virtual o usuário tem controle total da experiência dentro do mundo virtual 3D.

No artigo de Silva, Gruber, Marcelino (2012) (artigo 8 da Tabela 1) mostra a possibilidade de realizar a atividade prática completamente remotamente, via redes celulares $3 G$ ou através da internet para acessar o experimento e realizar as experiências necessárias para compreender plenamente as teorias da modulação por largura de pulso (MLP) (inglês PWM). Este Artigo é uma nova iniciativa para experiências remotas do mundo de diferentes especialidades de engenharia, envolvendo as redes celulares que estão em rápida expansão neste momento.

Já o artigo de Gruber, Marcelino, Silva (2013) (artigo 3 da Tabela 1) trataram da tecnologia PLC tecnologia (Comunicação pela rede elétrica, ou seja, utiliza a rede elétrica existente como meio de transmissão de dados), como uma solução para o monitoramento remoto de parâmetros de temperatura de mini turbinas em bancada. Realizaram testes comparativos entre o PLC e tecnologia LAN em dois ambientes distintos: residencial e 
Janine De Lavechia, Juarez Bento da Silva, Fernando José Spanhol

Publicações científicas do laboratório de experimentações remota - RExLab: uma revisão sistemática:

industriais. Os dados obtidos neste estudo indicam resultados satisfatórios sobre a tecnologia PLC em relação à LAN, confirmando a possibilidade de usar esta tecnologia como uma alternativa aos sistemas de monitoramento on-line.

Outro artigo que não está relacionado com o ensino e aprendizagem de alunos é o artigo de Gruber et al (2011)(artigo 11 da Tabela 1) mostra o desenvolvimento de um modelo de plataforma que pode monitorar adquirir, armazenar e transmitir dados remotamente usando sensores, rede de telefonia celular $3 G$ e as redes sociais para ser aplicado ao projeto de pequena turbina eólica.

O último artigo da Tabela 1 é o do Marcelino et al. (2010) traz como objetivo a intenção de facilitar a construção, gestão e partilha de conhecimento entre professores e alunos, numa perspectiva global. Com representações sociais 3D para acessar materiais de aprendizagem de uma forma dinâmica e interativa, que é considerado para estar mais perto da realidade física experimentada por professores e alunos.

\section{CONSIDERAÇÕES FINAIS}

A instituição facilita o processo de ensino quando desenvolve a cultura de estimular o aprendizado, superando barreiras e incentivando a aproximação de diferentes grupos, melhorando a comunicação entre eles e o fluxo de informações. Quando surge uma nova cultura de compartilhamento, esta deve ser esclarecida e levada a todos os membros de uma organização para que ela ocorra efetivamente. Para que isso ocorra, todos os envolvidos precisam participar e se envolver para que o resultado seja o esperado (EMYDIO, 2010).

O RExLab é um exemplo, pois possui um número considerável de publicações disponibilizadas no seu portal (http://rexlab.ufsc.br/publications) sobre as pesquisas do grupo, que de alguma forma, estão compartilhando o conhecimento. Os artigos na sua grande maioria estão relacionados com o apoio em facilitar o ensino e aprendizagem de alunos da rede pública de ensino dos anos iniciais até os alunos de engenharia, com a utilização da experimentação remota aplicada a diversos tipos de tecnologias. Com esse aparato das experimentações remotas os professores têm uma capacidade de 
Janine De Lavechia, Juarez Bento da Silva, Fernando José Spanhol

Publicações científicas do laboratório de experimentações remota - RExLab: uma revisão sistemática:

desenvolver ainda mais o aluno, apresentando na prática o desenvolvimento de uma fórmula, por exemplo, e isso consequentemente levará a uma diminuição da taxa de reprovação de uma disciplina com um grau de dificuldade alto, exemplo a disciplina de física.

Com o propósito de pesquisar e identificar os estudos e publicações relacionados ao grupo de estudantes pertencentes ao RExLab da Universidade Federal de Santa Catarina, Campus Araranguá, utilizou-se a técnica de revisão sistemática, e realizou-se uma análise dos artigos com maiores citações, apresentando as NTICs utilizadas em cada pesquisa.

Foi possível analisar que o maior objetivo do RExLab é a realização da democratização e a disseminação do conhecimento através de seus experimentos. Pois são projetos que podem estar aplicados no ensino público (desde as séries iniciais até o ensino médio, graduação), pois a instituição precisa apenas do acesso a internet e um dispositivo móvel, e assim alunos e professores tem a possibilidade de construir novos conhecimentos e compartilhar essa experiência adquirida, de uma maneira mais rápida. Sem dúvida, o RExLab é uma proposta de levar o conhecimento de uma forma prática, e de grande abrangência.

Porém, há um ponto que precisa ser verificado, pois a grande maioria das escolas da rede pública não possui internet disponível ao uso dos alunos, ou por falta de investimento, ou por falta de manutenção, onde apenas a parte administrativa possui o acesso. Isso é uma questão muito intrigante, pois no contexto que estamos inseridos, na era da informação e conhecimento, o acesso a internet é um bem primordial, e que deva estar disponível, para que seja possível a construção de novos conhecimentos.

Para trabalhos futuros sugere-se uma pesquisa englobando todos os tipos de publicações da equipe e que esteja em outras bases de dados, para que assim possa ter um panorama sobre o que estão pesquisando, tendo assim um mapeamento, o que deverá auxiliar na gestão do conhecimento do laboratório futuramente. 
Janine De Lavechia, Juarez Bento da Silva, Fernando José Spanhol

Publicações científicas do laboratório de experimentações remota - RExLab: uma revisão sistemática:

\section{REFERÊNCIAS}

BROFMAN, P. R. A importância das publicações científicas. Cogitare Enfermagem, v. 17, n. 3, p. 419-21, 2012.

CAPELLINI, S. A.; CONRADO, T. L. B. C.. Desempenho de escolares com e sem dificuldades de aprendizagem de ensino particular em habilidade fonológica, nomeação rápida, leitura e escrita. Cefac, São Paulo, p.1-11, ago. 2008.

CARDOSO, D. C.; TAKAHASHI, E. K.. Experimentação remota em atividades de ensino formal: um estudo a partir de periódicos Qualis A. Revista Brasileira de Pesquisa em Educação em Ciências, Uberlândia, v. 11, n. 3, p.185-208, mar. 2011. Disponível em: <http://nutec.ufu.br/experimentos/artigos/RBPEC113-2011.pdf>. Acesso em: 25 mai. 2016.

CRISPIM JUNIOR, J. TIC vs NTIC. 2013. Disponível em: <http://www.josecrispim.pt/artigos/conceitos/conc_art/01_tic_ntic.html>. Acesso em: 30 mai. 2016.

CRUZ, T. Sistema, organização e métodos: estudo integrado das novas tecnologias da informação. São Paulo: Atlas, 1998.

EMYDIO, M. M.; ROCHA, R. F. Gestão do conhecimento na área educacional: a tecnologia como instrumento facilitador. In: SIMPÓSIO DE EXCELÊNCIA EM GESTÃO E TECNOLOGIA, 9, 2012, Resende. Anais ... Resende: AEDB, 2012. Disponível em:

http://www.fatecgarca.edu.br/revista/Volume2/Artigo 7 Volume 2.pdf.

GUEDES, V. L. S.; BORSCHIVER, S. Bibliometria: uma ferramenta estatística para a gestão da informação e do conhecimento, em sistemas de informação, de comunicação e de avaliação científica e tecnológica. In: ENCONTRO NACIONAL DE CIÊNCIA DA INFORMAÇÃO, 6., 2005, Salvador. Anais... Salvador: ICI/UFBA, 2005 Disponível em:

< https://www.aedb.br/seget/arquivos/artigos12/31316263.pdf>. Acesso em: 21 abr. 2016.

GOMES, M.; DOURADO, R. Redes da informação e comunicação [exercício de aula]. Disponível em: <https://issuu.com/marisagomes8/docs/2013-04-11__ft2__16-marisa_gomes_21-rafael_doura>. 2013. Acesso em: 02 jun. 2016.

GRUBER, V. MARCELINO, R.; SILVA, J. B.Technology PLC - Power Line Communication, Used in Monitoring Systems Online. International Journal Of Online Engineering (ijoe), v. 9, n. 1, p.22-25, jan. 2013. 
Janine De Lavechia, Juarez Bento da Silva, Fernando José Spanhol

Publicações científicas do laboratório de experimentações remota - RExLab: uma revisão sistemática:

GRUBER, V.; SCHAEFFER, L.; SILVA, J. B.; RESTIVO, T. M. - Model for remote data acquisition and monitoring integrating social media, NTIC's and 3G cell phone networks applied to monitoring small wind turbine.

JournalofTelecommunications, v. 7, 2011.

LATTES, Diretório dos Grupos de Pesquisa no Brasil. RExLab - Laboratório de Experimentação Remota. Disponível em:

<http://dgp.cnpq.br/dgp/espelhogrupo/9180006433721466>. Acesso em: 26 mai. 2016.

LIMA, J. P. C.; ROCHADEL, W.; SILVA, J. B. Utilização da Experimentação Remota Móvel em Disciplina de Física do Ensino Médio. International Conference on Interactive Computer aided Blended Learning. 2013.

MACALHA. HTML5. Disponívelem: <https://developer.mozilla.org/ptBR/docs/Web/HTML/HTML5>. Acessoem: 05 jun. 2016.

MARCELINO, Roderval et al. 3D virtual worlds using open source platform and integrated remote experimentation. Remote Engineering And Virtual

Instrumentation, Florianópolis, v. 0, n. 0, p.1-2, jul. 2012.

MARCELINO, R. et al. Extended Immersive Learning Environment: A Hybrid Remote/Virtual Laboratory. International journal of online engineering, v. 6 , p. 1-6, 2010.

ORTIZ, C. E. Introdução ao jQuery Mobile. Disponível em: $<$ http://www.ibm.com/developerworks/br/library/wa-jquerymobileupdate/>. Acesso em: 05 jun. 2016.

ROCHADEL, W.et al. Extending Access to Remote Labs from Mobile Devices in Educational Contexts. International Journal Of Online Engineering (ijoe), v. 9 , n. 3, p.9-13, jan. 2013.

ROCHADEL, Willian et al., Utilization of Remote Experimentation in Mobile Devices for Education, 2012 IEEE Global Engineering Education Conference (EDUCON), pp. 1-6, Apr. 2012.

SILVA, R. S.. Análise do uso de laboratórios de experimentação remota como ferramenta de apoio à aprendizagem. 2015. 94 f. Dissertação (Mestrado) Curso de Programa de Pós-graduação em Modelagem Computacional de Sistemas, Universidade Federal do Tocantins, Palmas, 2015.

SILVA, J. B. da et al. Adaptation Model of Mobile Remote Experimentation for Elementary Schools. leee Revista Iberoamericana de Tecnologias Del Aprendizaje, v. 9, n. 1, p.28-32, fev. 2014. 
Janine De Lavechia, Juarez Bento da Silva, Fernando José Spanhol

Publicações científicas do laboratório de experimentações remota - RExLab: uma revisão sistemática:

SILVA, A. R. L.; SPANHOL, F. J.. Contribuições da criatividade em diferentes áreas do conhecimento: do pensar criativo à prática inovadora na EaD. São Paulo: Pimenta Cultural, 2013.

SILVA, Juarez B. da et al. Uso de dispositivos móveis para acesso a Experimentos Remotos na Educação Básica. Vaep-rita, v. 1, n. 2, p.129-134, jun. 2013.

SILVA, Juarez Bento da et al. Utilization of NICTs applied to mobile devices. IEEE Revista Iberoamericana de Tecnologias Del Aprendizaje, v. 8, n. 3, p.97-102, ago. 2013.

SILVA, J. B.; GRUBER, V.; MARCELINO, R. New Technologies for Information and communication, PWM remote experimenting and $3 G$ Networks as Teaching Support. International Journal of Engineering Pedagogy, v. 2, n. 1, p. 17-22, 2012.

SILVA, J. B.; ROCHADEL, W.; MARCELINO, R. Utilização de NTIC's aplicadas a dispositivos móveis. IEEE-RITA, v. 7, n. 3, p. 149-154, 2012.

SILVA, J. B. A utilização da experimentação remota como suporte para ambientes colaborativos de aprendizagem. 2006. $196 \mathrm{f}$. Tese (Doutorado) Curso de Programa de Pós-graduação em Engenharia de Gestão do Conhecimento, Universidade Federal de Santa Catarina, Florianópolis, 2006.

SIMÃO, J. P. S. et al. Utilização de experimentação remota móvel no ensino médio. Renote: novas tecnologias na educação, Porto Alegre, v. 11, n. 1, p.1-11, jul. 2013. Disponível em:

<http://seer.ufrgs.br/index.php/renote/article/view/41701/26452>. Acesso em: 26 mai. 2016.

TAKAHASHI, T. Sociedade da Informação no Brasil: Livro verde. Brasília: Ministério da Ciência e Tecnologia, 2000.

\section{Title}

Scientific publications laboratory of remote experimentation - RExLab: a systematic review

\section{Abstract:}

Introduction: The Information and Communication Technologies TICs are allowing day by day the improvement in all levels of the institutions, providing in a general context a greater communication, and enabling the creation of new knowledge. And with the emergence of the Remote Experimentation Laboratory - RExLab, which allows the student to perform experiments, makes possible the construction of new knowledge.

Objective: Identify and present the NICT (New Information and Communication Technologies) studied in the laboratory and explained in the publications of the members linked RExLab. 
Janine De Lavechia, Juarez Bento da Silva, Fernando José Spanhol

Publicações científicas do laboratório de experimentações remota - RExLab: uma revisão sistemática:

Methodology: In this article a systematic research was done on the articles published in RExLab, and located at the Federal University of Santa Catarina, Araranguá. And the articles with the highest citations, the NICT used in each research were presented.

Result: It was possible to identify that a great majority of articles published was related to questions of remote experimentation through the usage of mobile devices, and today it allows almost all the students to have a great access to it.

Conclusion: It was possible to analyze that the greatest objective of published research is the achievement of democratization and the dissemination of knowledge through their experiments. These are projects that can be applied at all levels of education and also in public and private schools.With this, there are many ICTs that are inserted in the projects and that they have as purpose the aid to the education.

Keywords: Publications. RExLab. ICT.

\section{Titulo}

Las publicaciones científicas de laboratorio de eperimentación a distancia RExLab: una revisión sistemática

\section{Resumen:}

Introducción:Tecnologías de Información y Comunicación TIC son cada día permitiendo la mejora en todos los niveles de las instituciones, proporcionando en un contexto general una mayor comunicación, y permitiendo así la creación de nuevos conocimientos.Y con el surgimiento del Laboratorio de Experimentación Remota RExLab, que posibilita al alumno realizar experimentos, viabiliza la construcción de nuevos conocimientos.

Objetivo: identificar y presentar las NTIC (nuevas tecnologías informáticas y de comunicación) estudiaron en el laboratorio y se explican en las publicaciones de los miembros vinculados al RExLab

Metodología: En este artículo se realizó una investigación sistemática sobre los artículos publicados en el RExLab, y situado en la Universidad Federal de Santa Catarina, Araranguá. Y los artículos con mayores citas, fueron presentadas las NTICs utilizadas en cada investigación.

Resultado: Es posible identificar que una gran mayoría de los artículos publicados estaban relacionados en cuestiones de experimentación remota a través de dispositivos móviles, y que hoy es de gran acceso a casi todos los alumnos.

Conclusión: Fue posible analizar que el mayor objetivo de las investigaciones publicadas es la realización de la democratización y la diseminación del conocimiento a través de sus experimentos. Porque son proyectos que pueden estar aplicados en todos los niveles de enseñanza y también tanto en escuelas públicas como en escuelas privadas. Con ello, hay muchas TIC que están insertadas en los proyectos y que tienen como propósito el auxilio a la educación.

Palabras clave: Publicaciones. RExLab. TIC.

Recebido em: 01.09.2016

Aceito em: 13.12.2017 